Objectives: To investigate the effect of empathy nursing on the life quality of patients with Systemic Lupus Erythematosus (SLE).

Methods: 120hospitalized patients with SLE admitted from January 2014 to December 2016 were divided into the control group and the experimental group for 60 people in each randomly. The control group was given routine care and the experimental group was given additional empathy care for 6 months. The World Health Organization Quality of Life (WHOQOL-BREF) Chinese version was evaluated on the 2nd day of hospitalization and 6 months after discharge respectively.

Results: Before intervention, the life quality of the two groups was poor. The scores of the control group and the experimental group in each field had no statistic difference $(44.13 \pm 16.72$ vs $44.08 \pm 17.33$ in physiology, $51.13 \pm 14.38$ vs $52.01 \pm 13.87$ in psychology, $58.12 \pm 15.33$ vs $56.71 \pm 8.12$ in social relation, $54.93 \pm 13.2$ vs $55.33 \pm 11.78$ in environment and $52.52 \pm 15.6 v s 52.03 \pm 13.44$ overall), $(P>0.05)$. After the intervention, the scores of the WHOQOL-BREF scale in the two groups were improved to different extents $(P<0.05) \quad(59.33 \pm 13.76$ vs $66.77 \pm 16.21$ in physiology, $57.43 \pm 7.88$ vs $64.55 \pm 11.76$ in psychology, $65.22+13.34$ vs $72.11+8.12$ in social relation and the overall scores were $59.95 \pm 14.32$ vs $67.89 \pm 6.42$ ). The scores of the four dimensions in physiology, psychology, social relations and environment were significantly different from those before the intervention $(P<0.05)$. The improvement of the scores in physiology, psychology, social relations in the experimental group was more obvious than the control group $(\mathrm{P}<0.05)$

Conclusions: Empathy nursing can obviously improve the life quality of SLE patients, and it is worthy to be popularized.

References:

[1] Empathy - can it be taught? Jeffrey D, Downie R.J R Coll Physicians Edinb. 2016 Jun;46(2):107-112.

[2] Communication Needs of Patients with Breast Cancer: A Qualitative Study. Khoshnazar TA, Rassouli M, Akbari ME, Lotfi-Kashani F, Momenzadeh S, Rejeh N, Mohseny M. Indian J Palliat Care. 2016 Oct-Dec;22(4):402-409.

Disclosure of Interest: None declared

DOI: 10.1136/annrheumdis-2017-eular.3096

\section{AB0546 CLINICOPATHOLOGICAL CHARACTERISTICS OF SJÖGREN'S SYNDROME IN THE PRESENCE OR ABSENCE OF OBJECTIVE SICCA SYMPTOMS}

Y. Suzuki ${ }^{1,2}$, H. Fujii ${ }^{1}$, K. Yamada ${ }^{1}$, M. Kawano ${ }^{1} .{ }^{1}$ Division of Rheumatology, Department of Internal Medicine, Kanazawa University Graduate School of Medicine; ${ }^{2}$ Division of Nephrology and Rheumatology, Department of Internal Medicine, Ishikawa Prefectural Central Hospital, Kanazawa, Japan

Background: Sjögren's syndrome (SS) is generally diagnosed on the basis of objective criteria, including xerophthalmia, xerostomia, autoantibodies, and labial salivary gland biopsy. Patients without objective sicca symptoms (non-sicca SS) require a biopsy. For such patients, we should evaluate pretest probability using parameters other than sicca symptoms before performing an invasive biopsy. To assess pretest probability, data on clinicopathological characteristics of non-sicca SS are needed.

Objectives: This study aimed to analyze the clinicopathological features of non-sicca SS. Epidemiological data, antibody profiles, organ involvement, and labial salivary gland biopsy results in non-sicca SS patients were compared with those in SS patients with objective sicca symptoms (sicca SS).

Methods: We selected 103 patients with primary SS who met Japanese or American College of Rheumatology criteria; those whose results exceeded the focus score by 1 underwent salivary gland biopsy. Objective xerophthalmia was evaluated with the Schirmer's test, and objective xerostomia with the Saxon's test. Seventeen patients were excluded because neither test was performed. Sicca SS was defined as a positive Schirmer's and/or Saxon's test result. Clinical and laboratory data were compared in 70 sicca SS and 16 non-sicca SS patients.

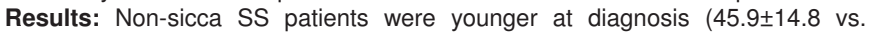
$61.4 \pm 15.1$ years, $p<0.001)$, had a shorter disease duration $(1.1 \pm 1.5$ vs. $6.9 \pm 8.9$ years, $\mathrm{p}<0.001$ ), and had a higher rate of positive anti-SS-A/Ro antibody $(100$ vs. $74.3 \%, p=0.023$ ), and a lower rate of positive anti-centromere antibody ( 6.3 vs. $44.3 \%, p=0.005$ ). Subjective xerophthalmia and xerostomia rates were similar between the groups, but fewer non-sicca SS patients had sicca symptoms as chief complaints ( 18.8 vs. $58.6 \%, p=0.004)$. There were no significant differences in focus score, leukocyte and lymphocyte counts, serum IgG levels, and positive rheumatoid factor and antinuclear antibody levels. The maximum European League Against Rheumatism Sjögren's Syndrome Disease Activity Index (ESSDAl) score during follow-up showed no significant difference (3.34 44.27 in non-sicca SS vs. $3.83 \pm 4.68$ in sicca SS, $p=0.30$ ). However, more non-sicca SS patients had ESSDAl scores $\geqq 1$ ( 100 vs. $71.4 \%, p=0.015)$, a positive correlation with the biological domain of the ESSDAI ( 87.5 vs $58.6 \%, p=0.03$ ), and articular symptoms ( 37.5 vs $8.6 \%, p=0.003$ ).

Conclusions: Non-sicca SS patients were younger, had shorter disease duration, and a higher rate of positive correlation with the biological and articular domains of the ESSDAI. Moreover, all non-sicca SS patients had ESSDAI scores $\geq 1$. When we diagnose SS patients without objective sicca symptoms, we should assess age, disease duration, and extraglandular organ involvement before performing labial salivary gland biopsy.

Disclosure of Interest: None declared
DOI: 10.1136/annrheumdis-2017-eular.5177

\section{AB0547 THE PREVALENCE AND THE RISK FACTOR OF HYPERTENSION AND DYSLIPIDEMIA IN SYSTEMATIC LUPUS ERYTHEMATOSUS PATIENTS: EXPLORATORY RESEARCH}

Y. Miura ${ }^{1}$, M. Saito ${ }^{1}$, K.-E. Sada ${ }^{2}$, N. Yajima ${ }^{1} .{ }^{1}$ Division of Rheumatology, Department of Internal Medicine, Showa University School of Medicine, Shinagawa-ku, Tokyo; ${ }^{2}$ Department of Nephrology, Rheumatology, Endocrinology and Metabolism, Okayama University Graduate School of Medicine, Dentistry and Pharmaceutical Sciences, Okayama City, Japan

Background: Hypertension (HT) and dyslipidemia (DL) are the risk factors for all-cause mortality, cardiovascular and cerebrovascular disease, and end-stage renal disease in SLE patients ${ }^{1}$ ). Neither disease activity nor chronic damage were associated with the metabolic syndrome in SLE patients ${ }^{2)}$ and there were few reports about the risk factors of HT and DL in Japanese SLE patients.

Objectives: We aimed to describe a prevalence of $\mathrm{HT}$ and $\mathrm{DL}$ and to identify the risk factor of $H T$ and DL in Japanese SLE patients.

Methods: All SLE patients visited at Showa University Hospital and Okayama University Hospital from January 2016 to September 2016, were enrolled in a cross-sectional study. SLE patients who satisfied American College of Rheumatology (ACR) criteria were included. HT was defined as usage of anti-HT drugs and DL was defined as usage of anti-DL drugs. We performed descriptive statistics and binomial logistic regression analysis to identify the risk factors of $\mathrm{HT}$ and DL. Variables considered possible risk factors were BMI, drinking status, smoking status (current smoking), current daily dose of glucocorticoids, past maximum dose of glucocorticoids, lupus nephritis, Systemic Lupus Erythematosus Disease Activity Index 2000 (SLEDAI-2K), and Systemic Lupus International Collaborating Clinics/American College of Rheumatology Damage Index (SLICC/ACR-DI).

Results: In total, 244 participants were enrolled. The mean age was $46.2 \pm 15.3$ years, and $222(91 \%)$ were female. The mean current daily dosage of glucocorticoids was $6.7 \pm 5.9 \mathrm{mg}$, and the mean SLEDAI- $2 \mathrm{~K}$ was $5.0 \pm 5.2$ and the mean SLICC/ACR-DI was 1.3 \pm 1.7 . The prevalence of HT and DL were $29.1 \%(71 / 244)$ and $22.1 \%$ (54/244). Both HT and DL were confirmed in 11.9\% (29/244) patients. On binomial logistic regression analysis, BMI (regression coefficients $(\beta)=-0.095$; $95 \%$ confidential interval $(\mathrm{Cl})=-0.173$ to -0.020$)$, drinking status $(\beta=0.443 ; 95 \%$ $\mathrm{Cl}=0.000$ to 0.879$)$, past maximum dosage of glucocorticoids $(\beta=-0.018 ; 95 \% \mathrm{Cl}$ $=-0.036$ to -0.004$)$ and lupus nephritis $(\beta=-0.727 ; 95 \% \mathrm{Cl}=0.230$ to 1.241$)$ were identified as the significant independent risk factors of $\mathrm{HT}$. On the other hand, only age $(\beta=-0.030 ; 95 \% \mathrm{Cl}=-0.055$ to -0.006$)$ was identified as the independent risk factor of $D L$. There was no independent risk factor of having both $D L$ and $H T$. Conclusions: Our results could help to identify patients at higher risk of HT and DL.

\section{References:}

[1] Hsin-Hui Yu. et al. Statin reduces mortality and morbidity in systemic lupus erythematosus patients with hyperlipidemia: A nationwide population-based cohort study. Atherosclerosis. 2015;243:11-18.

[2] Cecilia P Chung. Et al. High prevalence of the metabolic syndrome in patients with systemic lupus erythematosus: association with disease characteristics and cardiovascular risk factors. Ann Rheum Dis. 2007;66:208-214.

Disclosure of Interest: None declared

DOI: 10.1136/annrheumdis-2017-eular.2875

\section{AB0548 CIRCULATING PROLACTIN LEVEL IN SYSTEMIC LUPUS ERYTHEMATOSUS AND ITS CORRELATION WITH DISEASE ACTIVITY: A META-ANALYSIS}

Y.H. Lee, Y.H. Seo. Rheumatology, Korea University Medicial Center, Seoul, Korea, Republic Of

Background: Prolactin has an immune stimulatory effect and may promote autoimmunity by encouraging the development of antigen presenting cells expressing MHC class II and co-stimulatory molecules and modulating IFN- $\gamma$ secretion.

Objectives: This study aimed to evaluate the relationship between circulating prolactin level and systemic lupus erythematosus (SLE), and to establish a correlation between plasma/serum prolactin levels and SLE activity.

Methods: We performed a literature search for studies that examined prolactin status in SLE patients and controls, and the relationship between circulating (serum or plasma) prolactin levels and SLE using PUBMED, EMBASE, and Cochrane databases. We conducted a meta-analysis comparing the plasma/serum prolactin levels in patients with SLE to controls, and examined correlation coefficients between circulating prolactin level and SLE disease activity.

Results: Twenty-five studies with a total of 1,056 SLE patients and 426 controls were included. Prolactin levels were significantly higher overall in the SLE group than in the control group $\left(\mathrm{SMD}=0.987,95 \% \mathrm{Cl}=0.512-1.463, p=4.7 \times 10^{-5}\right)$. Stratification by ethnicity showed significantly elevated prolactin levels in the SLE group in Asian, Latin American, and mixed populations (SMD $=0.813$, $95 \% \mathrm{Cl}=0.137-1.490, p=0.018 ; \mathrm{SMD}=0.981,95 \% \mathrm{Cl}=0.307-1.655, p$ $=0.004 ; \mathrm{SMD}=1.469,95 \% \mathrm{Cl}=0.443-2.495, p=0.005$, respectively), but not in the European population. Meta-analysis of correlation coefficients showed 